\title{
Takotsubo cardiomyopathy complicated by cardiac tamponade
}

\author{
E. ter Bals • D. A. M. Odekerken • G. A. Somsen
}

Published online: 7 August 2013

(C) The Author(s) 2013. This article is published with open access at Springerlink.com

\begin{abstract}
We describe a 76-year-old patient with takotsubo cardiomyopathy complicated by cardiac tamponade. Pericardial effusion in takotsubo cardiomyopthy is common but a cardiac tamponade is very rare. The use of anticoagulants may increase the risk of pericardial effusion and should be considered with care.
\end{abstract}

Keywords Takotsubo cardiomyopathy · Tamponade · Pericardial effusion · Cardioembolic complications . Anticoagulation

\section{Introduction}

Takotsubo cardiomyopathy is a syndrome with transient systolic dysfunction of the left ventricle in the absence of significant coronary artery obstruction. The contractile function of the mid and apical segments of the left ventricle is classically depressed and there is hyperkinesis of the basal segments. This leads to the typical apical ballooning of the left ventricle. Takotsubo cardiomyopathy is much more common in women than men and is frequently triggered by emotional or physical stress. It has even been described after pacemaker implantation. The clinical presentation mimics acute myocardial infarction. Patients present most commonly with chest pain, electrocardiographic abnormalities such as ST-segment elevation or T-wave inversion and elevated cardiac biomarkers. The

E. ter Bals $(\bowtie) \cdot G$. A. Somsen

Onze Lieve Vrouwe Gasthuis, Oosterpark 9, 1091 AC Amsterdam,

the Netherlands

e-mail: edske.terbals@gmail.com

D. A. M. Odekerken

Spaarne Ziekenhuis, Spaarnepoort 1, 2134 TM Hoofddorp,

the Netherlands left ventricular function usually recovers within days to weeks. Treatment of takotsubo cardiomyopathy is supportive to prevent heart failure. Complications include acute heart failure, tachyarrhythmias, bradyarrhythmias, mitral regurgitation, cardiogenic shock, transient left ventricular outflow tract (LVOT) obstruction, formation of a left ventricular thrombus, cardioembolic events, left ventricular free-wall rupture and death [1-5].

\section{Case}

A 76-year-old female presented to the coronary care unit with chest pain and an ECG suggestive for an inferolateral myocardial infarction (Fig. 1). Her medical history consisted of hypertension, surgery to her back and macular degeneration. She had never smoked and had two brothers with coronary artery disease before the age of 60 . This was the first time she had experienced chest pain. It started at the moment she was worrying about loss of eyesight due to macular degeneration.

A coronary angiogram was performed immediately and was normal. The left ventricular angiogram showed apical ballooning (Fig. 2). Echocardiography showed apical dyskinesia and no other abnormalities. These findings confirmed the diagnosis: takotsubo cardiomyopathy.

A beta blocker, ACE inhibitor and oral anticoagulation, to prevent apical thrombus formation, were started. In the following days the patient developed atrial fibrillation and she had recurrent chest and back pain. Analgesics were started. On the third day her blood pressure dropped and the diuresis decreased. Echocardiography revealed a pericardial effusion of up to $16 \mathrm{~mm}$, with diastolic collapse of the right ventricle and $>25 \%$ transmitral flow variation with respiration which is indicative for cardiac tamponade (Fig. 3). CT angiography ruled out an aortic dissection. The oral anticoagulation was 


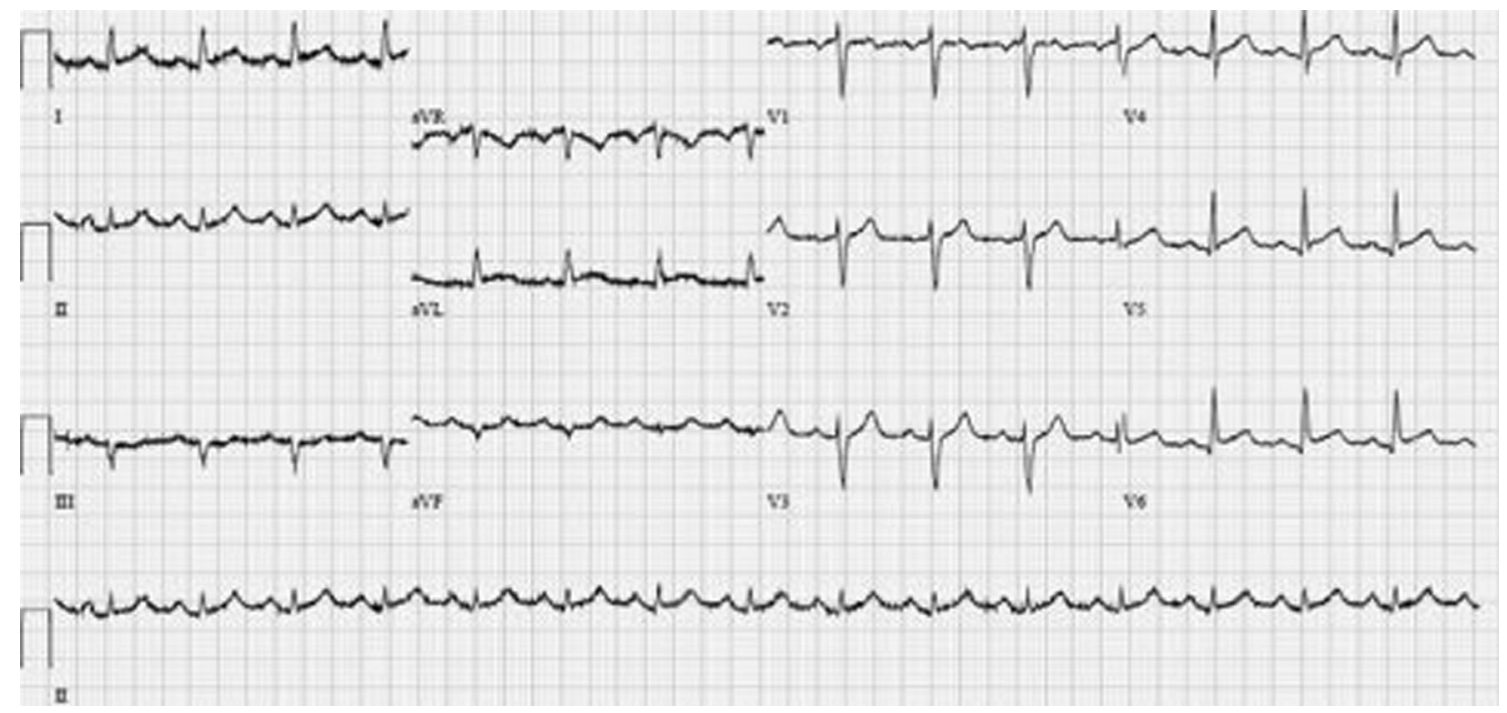

Fig. 1 ECG at presentation
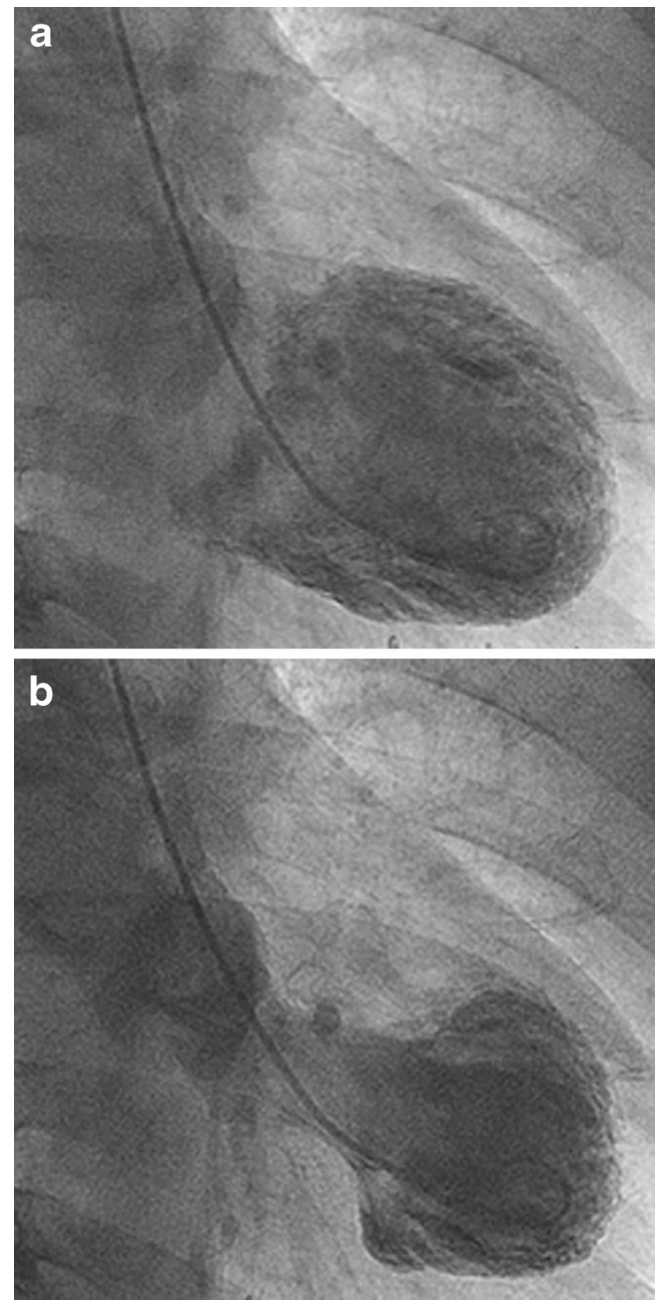

Fig. 2 Left ventricular angiogram a diastolic b systolic stopped and the INR of 2.6 was antagonised with vitamin $\mathrm{K}$. Pericardiocentesis was performed. After draining $120 \mathrm{cc}$ bloody fluid, there was an immediate improvement of the haemodynamics and diuresis.

\section{Discussion}

The exact pathogenesis of takotsubo cardiomyopathy is not well understood. Catecholamine excess, coronary artery spasm, microvascular dysfunction, dynamic mid-cavity or LVOT obstruction, inflammation and regional myocarditis may play a role $[3,6,7]$.

Inflammation extending to the pericardium may cause pericardial effusion. An MRI study showed that pericardial effusion is common (62\%) in takotsubo cardiomyopathy [7].

Cardiac tamponade in takotsubo cardiomyopathy, however is very rare. In 2010, one case was described by Yeh et al. in Circulation [8]. So far this complication has only been described in a total number of three cases.

The use of anticoagulation may increase the risk of pericardial effusion. Treatment with anticoagulants is recommended although there are no clear guidelines whether or not to start anticoagulation in takotsubo cardiomyopathy.

The incidence of left ventricular thrombus formation and cardioembolic events in takotsubo cardiomyopathy is $5 \%$ and $1.6 \%$ respectively. Of the cardioembolic events, around $75 \%$ are an ischaemic brain event. Not all patients with cardioembolic events show a left ventricular thrombus on their first echocardiography [9]. An ischaemic brain event may cause irreversible damage while pericardial effusion can be drained. 

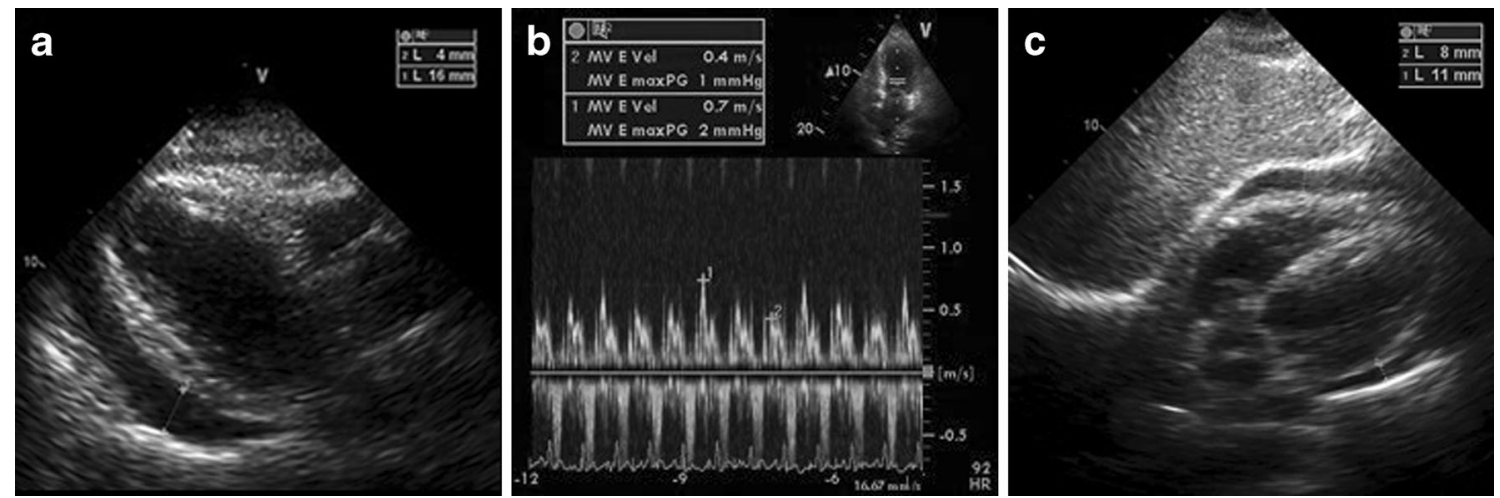

Fig. 3 Echocardiography a parasternal long axis $\mathbf{b}$ transmitral flow $\mathbf{c}$ subcostal view

Treatment with anticoagulants should therefore be considered at the moment of presentation to the hospital, irrespective of the presence of a left ventricular thrombus. The use of anticoagulants must of course be weighed against a patient's individual bleeding risk. Anticoagulants can be stopped after recovery of the left ventricular function.

\section{Conclusion}

Although takotsubo cardiomyopathy complicated by cardiac tamponade is very rare, echocardiography should be performed in case of haemodynamic deterioration to exclude pericardial effusion. The use of anticoagulation in these patients should be considered with care.

\section{Funding None.}

\section{Conflict of interests None declared.}

Open Access This article is distributed under the terms of the Creative Commons Attribution License which permits any use, distribution, and reproduction in any medium, provided the original author(s) and the source are credited.

\section{References}

1. Bybee KA, Kara T, Prasad A, et al. Systematic review: transient left ventricular apical ballooning: a syndrome that mimics ST-segment elevation myocardial infarction. Ann Intern Med. 2004;141:858-65.

2. Sharkey SW, Lesser JR, Zenovich AG, et al. Acute and reversible cardiomyopathy provoked by stress in women from the United States. Circulation. 2005;111:472-79.

3. Gianni M, Dentali F, Grandi AM, et al. Apical ballooning syndrome or takotsubo cardiomyopathy: a systematic review. Eur Heart J. 2006;27:1523-29.

4. Prasad A, Lerman A, Rihal CS. Apical balloonining syndrome (Takotsubo or stress cardiomyopathy): a mimic of acute myocardial infarction. Am Heart J. 2008;155:408-17.

5. Postema PG, Wiersma JJ, van der Bilt IAC. Takotsubo cardiomyopathy shortly following pacemaker implantation - case report and review of the literature. Neth Heart J. 2012. doi:10.1007/s12471-012-0320-8. Oct 11.

6. Akashi YJ, Goldstein DS, Barbaro G, et al. Takotsubo cardiomyopathy: a new form of acute, reversible heart failure. Circulation. 2008;118:2754-62.

7. Eitel I, Lücke C, Grothoff M, et al. Inflammation in takotsubo cardiomyopathy: insights from cardiovascular magnetic resonance imaging. Eur Radiol. 2010;20:422-31.

8. Yeh RW, Yu PB, Douglas E, et al. Takotsubo cardiomyopathy complicated by cardiac tamponade. Classic hemodynamic findings with a new disease. Circulation. 2010;122:1239-41.

9. de Gregorio C. Cardioembolic outcomes in stress-related cardiomyopathy complicated by left ventricular thrombus: a systematic review of 26 clinical studies. Int J Car. 2010;141:11-7. 